\title{
FORMATION CONTINUE : INDICATIONS POUR L'ENSEIGNEMENT À DISTANCE DES SCIENCES BIOLOGIQUES AU PRIMAIRE ET AU SECONDAIRE
}

\section{ARTICLE ORIGINAL}

DENDASCK, Carla Viana1', OLIVEIRA, Euzébio de ${ }^{2}$, FECURY, Amanda Alves ${ }^{3}$, DIAS, Claudio Alberto Gellis de Mattos ${ }^{4}$

DENDASCK, Carla Viana. Et al. Formation continue : indications pour l'enseignement à distance des sciences biologiques au primaire et au secondaire. Revista Científica Multidisciplinar Núcleo do Conhecimento. An 06, Ed. 08, vol. 06, p. 171 à 186. Août 2021. ISSN : 2448-0959, Lien d'accès: https://www.nucleodoconhecimento.com.br/education-fr/science-biologie, $\quad$ DOI: 10.32749/nucleodoconhecimento.com.br/education-fr/science-biologie

\section{RÉSUMÉ}

Bien que les discussions sur la possibilité d'une éducation en ligne à l'école primaire et secondaire au Brésil soient entrées dans le sillage du débat théorique, il existe encore une grande résistance. Cependant, le contexte de la pandémie n'a apporté aucune alternative, conduisant des milliers d'étudiants à l'isolement social pendant plus d'un an. Ce contexte a amené les enseignants du primaire et du secondaire à

\footnotetext{
${ }^{1}$ Théologien, Ph.D. en psychanalyse clinique. II travaille depuis 15 ans avec la méthodologie scientifique (méthode de recherche) dans le cadre de l'orientation de production scientifique des étudiants à la maîtrise et au doctorat. Spécialiste en études de marché et en recherche axée sur la santé. Doctorant en Communication et Sémiotique (PUC SP).

${ }^{2}$ Biologiste, Ph.D. en maladies tropicales, professeur et chercheur du cours d'éducation physique, Université fédérale du Pará (UFPA).

${ }^{3}$ Biomédical, Docteur en maladies tropicales, professeur et chercheur du cours de médecine du campus de Macapá, Université fédérale d'Amapá (UNIFAP), pro-recteur de la recherche et des études supérieures (PROPESPG) de l'Université fédérale d'Amapá (UNIFAP).

${ }^{4}$ Biologiste, Ph.D. en recherche théorique et comportementale, professeur et chercheur du cours de chimie de I'Institut d'éducation fondamentale, technique et technologique de l'Amapá (IFAP) et du Graduate Program in Professional and Technological Education (PROFEPT IFAP).
}

RC: 96092

Disponible en: https://www.nucleodoconhecimento.com.br/education-fr/science- 
s'adapter aux nouvelles technologies, démontrant ainsi la nécessité de se préparer à travailler dans l'enseignement à distance. La question guide de ce matériel était la suivante: comment les enseignants en sciences de la vie peuvent-ils se préparer et quels outils de base devraient-ils connaître pour préparer leurs cours en ligne? Ainsi, l'objectif général était de réaliser des indications que les enseignants devraient connaître pour préparer leurs cours en ligne. La méthodologie adoptée a été exploratoire par le biais d'une revue de la littérature. Les principales indications étaient que les enseignants doivent rechercher un contenu diversifié dans la préparation des classes pour adopter des stratégies de motivation avec leurs élèves, en adoptant un soin avec la question de la langue, de l'audio et de la lumière dans la production des classes. Enfin, il appartiendra à l'enseignant de reconnaître les différents outils et ressources tels que : Youtube, Instagram, Films, Jeux, et autres subventions, afin qu'il y ait une approximation entre l'enseignement de la biologie et la réalité de l'élève.

Mots-clés : Formation des enseignants, Enseignement à distance, Enseignement de la biologie.

\section{INTRODUCTION}

Depuis la fin de 2019, le monde a rencontré le virus SARS/COVID-19. Sa grande capacité de contagion, l'implication des organes vitaux, en particulier les poumons, et l'ignorance de la communauté scientifique, ont conduit les organisations internationales à décréter une situation pandémique mondiale, indiquant un retrait social immédiat. Cette mesure a eu un impact direct sur les organisations qui ont dû s'adapter à de nouvelles réalités, en utilisant, comme jamais auparavant, des moyens technologiques pour permettre la continuité de leurs fonctionnalités respectives. Selon Gonçalves et al. (2020), au Brésil, l'une des institutions les plus touchées a été les écoles, en particulier les écoles publiques, car, en plus de faire face à des problèmes concernant l'accessibilité des outils technologiques, leurs

RC: 96092

Disponible en: https://www.nucleodoconhecimento.com.br/education-fr/science- 
enseignants n'étaient pas préparés à cette réalité et beaucoup ont résisté à l'idée de l'éducation en ligne, en particulier au lycée et au primaire.

Lenzi et Santos (2021) ont fait allusion au fait que même avec les vaccins et le retour aux cours en face à face, il est important que les enseignants s'adaptent aux routines de l'enseignement à distance, en particulier dans ce qui a été préconisé par les enseignants comme: l'enseignement hybride, car ce contexte est déjà une réalité. C'est pourquoi cette étude doit fournir des subventions indicatives que les enseignants devraient prendre en compte lors de la préparation de leurs cours en ligne, en commençant par la question guide suivante: Comment les enseignants en sciences de la vie peuvent-ils se préparer et quels outils de base devraient-ils connaître pour préparer leurs cours en ligne? Ainsi, l'objectif général de cette étude sera : de réaliser des indications que les enseignants devraient connaître pour préparer leurs cours en ligne.

Des objectifs spécifiques seront envisagés: a) Démontrer les principales difficultés de l'enseignement en ligne des sciences biologiques et l'importance de l'utilisation des technologies; b) Rechercher des outils qui peuvent être intégrés à l'éducation en ligne; c) Fournir des indicateurs technologiques et des instructions qui devraient être pris en compte par l'enseignant de biologie. Ainsi, dans la base théorique, une recherche large a été utilisée dans Google Academic,en utilisant comme descripteurs: «Outils pour l'enseignement en ligne de la biologie »; «EAD Enseignement de la biologie »; «Enseignement hybride des sciences biologiques »; «Enseignement de la biologie hybride. » En tant que critères d'inclusion, il a été considéré comme des matériaux susceptibles de contribuer au problème et aux objectifs présentés ici. Vingt documents ont été sélectionnés, y compris des articles scientifiques, des livres et des manuels. En possession de ceux-ci, dans les résultats et les discussions, un tableau a été tenu avec des indications sur les outils de base et les considérations générales à adopter dans l'enseignement de la biologie en ligne.

RC: 96092

Disponible en: https://www.nucleodoconhecimento.com.br/education-fr/science- 


\section{L'IMPORTANCE DE LA FORMATION CONTINUE DES ENSEIGNANTS}

L'histoire de l'humanité est dirigée et constituée à partir de différentes perspectives sociales, politiques, scientifiques et économiques, et toutes ces dimensions sont affectées par les progrès technologiques (TREBIEN et al.,2020). Tout changement social et de système affecte directement le processus éducatif dans l'école et, par conséquent, l'institution scolaire doit suivre ces progrès (RODRIGUES; LIMA; VIANA, 2017). L'école doit s'adapter au nouveau modèle. Compte tenu de ce scénario, le professeur est fortement impliqué dans un processus qui implique des changements constants afin qu'il puisse relever les défis imposés par la société. En effet, nous vivons à l'ère de l'information et, par conséquent, la formation initiale des enseignants n'est plus une exigence suffisante pour garantir la maîtrise du contenu et la qualité pédagogique (JUNGES; KETZER; OLIVEIRA, 2018).

La formation continue admet les activités développées par les enseignants après la première formation. Cela se produit lorsque le professionnel entre dans la profession enseignante. II peut être individuel ou collectif (TREBIEN et al., 2020). II devient donc indispensable un processus de formation permanent et continu, enraciné dans une épistémologie de la pratique et de la vie quotidienne, pouvant subventionner une action éducative innovante et consciente (JUNGES; KETZER; OLIVEIRA, 2018). En raison de la pertinence de la continuité de la formation des enseignants, la Loi sur les lignes directrices et les bases de l'éducation (LDB) contient un chapitre qui met l'accent sur le sujet et fournit un soutien à cette formation: « [...] l'association entre les théories et les pratiques, y compris par le biais de la formation en cours d'emploi ", à la suite du paragraphe unique, met en évidence la « formation continue pour les professionnels sur leur lieu de travail » (TREBIEN et al., 2020, 94).

Compte tenu de l'augmentation et de l'amélioration des politiques visant la formation continue, principalement en raison du souci de la qualité de l'exercice

RC: 96092

Disponible en: https://www.nucleodoconhecimento.com.br/education-fr/science- 
d'enseignement, le thème a suscité de nouvelles actions. II est alors nécessaire de rejoindre un programme de formation continue bien conçu avec des objectifs bien structurés, comprenant non seulement les besoins de l'enseignant, mais aussi l'ensemble du système éducatif, impliquant les enseignants dans ce processus de planification, qui doit être participatif, collaboratif et doit admettre leurs besoins réels (RODRIGUES; LIMA; VIANA, 2017). Pour que leurs connaissances et leur pratique soient valorisées, l'enseignant, avec ses pairs, doit réfléchir et dialoguer, en aidant les autres à surmonter leurs conflits (FALSARELLA, 2004). De cette façon, la formation continue contribuera au développement de l'autonomie de l'enseignant et du dynamisme de sa pratique quotidienne :

[...] la formation continue en tant que proposition intentionnelle et planifiée, qui vise à changer l'éducateur par un processus réflexif, critique et créatif, il est conclu qu'elle devrait motiver l'enseignant à être un agent actif dans la recherche de sa propre pratique pédagogique, produisant des connaissances et intervenant dans la réalité (FALSARELLA, 2004, p. 50).

La formation continue de l'enseignant implique l'adoption d'une posture continue qui implique la réflexion, la recherche, l'action, la découverte, l'organisation, la fondation, la révision et la construction théorique. II ne s'agit pas seulement de l'apprentissage de nouvelles techniques pédagogiques ou des dernières innovations pédagogiques (FALSARELLA, 2004). En ce sens, le processus de formation permanente doit être basé sur la réalité de chaque école, et il est nécessaire que les rencontres pédagogiques et autres moments de formation, à l'intérieur et à l'extérieur de l'école, soient repensés chaque jour, afin de suivre les changements de la société elle-même (GADOTTI, 2008). Le rôle de l'enseignant dans ce contexte hautement variable et dynamique implique la contextualisation des contenus enseignés, de sorte que l'apprentissage devient significatif, ce qui nécessite un exercice constant de réflexion critique.

[...] la connaissance théorique proposition est donc articulée à la connaissance de la pratique, tout en se résignant et en étant resignifiée par eux. Le rôle de la théorie est d'offrir aux enseignants des

RC: 96092

Disponible en: https://www.nucleodoconhecimento.com.br/education-fr/science- 
perspectives d'analyses pour comprendre l'historique, le social, le culturel, l'organisationnel, et eux-mêmes en tant que professionnels, dans lesquels se déroule leur activité d'enseignement, pour intervenir en eux, les transformer (GHEDIN, 2012, p. 31).

Il est nécessaire que le professeur s'approprie la théorie afin qu'il puisse réfléchir et interpréter la pratique de l'enseignement en fonction des exigences de la société, ce qui implique des changements dans la pratique pédagogique, afin qu'il soit possible de renforcer l'autonomie et le dynamisme de l'enseignant (TREBIEN et al, 2020). La pratique pédagogique est le point de départ et d'arrivée du processus de formation continue et, en même temps, c'est un chemin qui permet la transformation de l'action de l'enseignant, et cherche ainsi le fondement théorique des subventions qui permettent le renforcement de la praxis de manière réflexive, dialogique et interactive (GHEDIN, 2012). II appartient donc à l'enseignant d'être prêt à innover, afin qu'il puisse répondre aux exigences de la société dans le domaine de l'enseignement, afin qu'il puisse transformer la pratique et la pratique professionnelle (RODRIGUES; LIMA; VIANA, 2017).

A partir du moment où l'on commence à réfléchir à la formation continue et à la manière dont elle doit s'adapter aux multiples avancées (y compris celles d'ordre technologique), une conscience intentionnelle de la qualité sociale de l'éducation et de la pratique pédagogique scolaire s'impose (JUNGES; KETZER; OLIVEIRA, 2018). Par conséquent, il est entendu qu'une éducation de qualité épistémologique et sociale prône un objectif fondamental : la nécessité d'une pratique pédagogique créative et stratégique est défendue, et, par conséquent, elle ne doit pas être façonnée par la simple répétition de pratiques existantes (GHEDIN , 2012). En ce sens, l'élaboration et l'application de politiques visant à la formation sont essentielles. De nos jours, celles axées sur la performance des technologies en classe sont fondamentales. C'est une réalité qui nécessite une manipulation prudente de la théorie et de la pratique dans l'enseignement (TREBIEN et al, 2020).

RC: 96092

Disponible en: https://www.nucleodoconhecimento.com.br/education-fr/science- 
Toutefois, il convient d'attirer l'attention sur un problème auquel est confronté le système éducatif brésilien. Au Brésil, historiquement, les modèles prédominants de formation continue ont privilégié une conception purement instrumentale du travail d'enseignement (FERREIRA; ALBUQUERQUE; LEAL, 2007). En ce sens, l'enseignant est amené à reproduire des techniques et, passivement, exécute les propositions déjà prêtes. On remarque que, dans de nombreux cas, les normes prescrivent un modèle d'enseignement qui dirige l'étudiant vers un apprentissage actif, participatif et autonome. Cependant, dans la pratique, il n'y a pas d'articulation de ces connaissances pédagogiques dans les cours de formation continue (TREBIEN et al., 2020). Cependant, la formation continue contribue à l'amélioration de l'enseignant et, par conséquent, à la qualité de l'enseignement. Ainsi, plusieurs connaissances de la pratique éducative deviennent nécessaires à la resignification de la pratique de l'enseignement, ce qui implique une recherche et un apprentissage constants de la part de l'enseignant.

\section{L'ENSEIGNEMENT À DISTANCE COMME OUTIL DE FORMATION CONTINUE}

L'utilisation des technologies et l'enseignement à distance est une réalité qui doit être intégrée par les enseignants, car la pandémie a provoqué une sorte de rupture des relations et, par conséquent, dans l'éducation. On estime que même avec le contrôle de la COVID, l'école devrait s'adapter à une autre réalité, sinon à un enseignement entièrement en ligne, du moins l'adoption par des modèles hybrides (LENZI et al., 2021). En ce qui concerne les difficultés de l'enseignement en ligne, Gonçalves et al. (2020) ont considéré la relation entre l'école et la famille fondamentale, car il sera à l'élève de suivre le développement de l'élève chez lui. Nicola et Paniz (2016) ajoutent que quelle que soit la technologie choisie, il est important que l'enseignant garde à l'esprit que la diversification des ressources technologiques éducatives sera essentielle à la réussite de l'enseignement de l'apprentissage.

RC: 96092

Disponible en: https://www.nucleodoconhecimento.com.br/education-fr/science- 
En outre, il est nécessaire de rechercher des environnements qui traduisent la réalité quotidienne et le besoin de la société dans laquelle l'étudiant est inséré (ARAÚJO et al. 2011).

[..] la « salle de classe », traduite en un espace virtuel où les leçons sont mises à disposition, ne suffit pas à assurer le résultat souhaité. Comme dans une école, de la manière la plus traditionnelle, il est nécessaire de considérer tous les agents impliqués. II faut dire aussi que même si l'on a une notion plus proche de la réalité et que l'on a l'idéal des participants au processus, cela ne suffit pas à assurer la réalisation des objectifs éducatifs proposés. En d'autres termes, il ne s'agit pas d'avoir les composantes d'une école et de les rassembler au même endroit. Ce que l'on veut avec le concept d'école, c'est l'intégration harmonique des pièces afin que le fonctionnement de cet engrenage présente le moins possible de bords (SOARES FILHO, 2013, p.418).

Olivetti et Periotto (2014), en interrogeant 88 enseignants, ont conclu que la formation continue est fondamentale pour l'efficacité de l'utilisation des technologies dans l'enseignement de la biologie, car la biologie seule a toujours nécessité des ressources autres que théoriques, telles que: laboratoires et terrain. En outre, l'enseignant doit garder à l'esprit que l'enseignement de la biologie est un outil de construction critique et sociale de la matière et, par conséquent, il est nécessaire d'utiliser toutes les ressources pour que l'élève ait une meilleure performance (LEITE et al., 2017), y compris l'adoption de la prise de conscience que l'étudiant doit comprendre la biologie dans sa réalité quotidienne (DURÉ et al., 2018).

Repenser, donc, l'enseignement de la biologie, Brandim et Nogueira (2018); Machado (2017), a mené une réflexion sur les pratiques employées et les possibilités des outils technologiques dans l'enseignement de la biologie, et souligne la nécessité pour l'enseignant de rechercher une formation constante pour améliorer le résultat de sa pratique professionnelle et son rôle social en tant qu'enseignant. En plus du contexte d'enseignement de l'apprentissage axé sur la matière enseignée, l'enseignant contemporain doit garder à l'esprit qu'il devient également un éducateur technologique, c'est-à-dire responsable d'enseigner à l'élève l'utilisation de la

RC: 96092

Disponible en: https://www.nucleodoconhecimento.com.br/education-fr/science- 
technologie de manière constructiviste, en indiquant les meilleures options d'utilisation pour la construction de la pensée critique et une meilleure absorption du contenu qui peut servir dans leur processus de formation, et, par conséquent, interférer dans le comportement numérique (ROCHA, 2013).

La virtualisation des systèmes éducatifs présuppose l'altération des modèles d'enseignement et des pratiques d'enseignement actuels, et donc la situation « oblige » l'enseignant à assumer de nouveaux rôles, communiquant ainsi par des moyens auxquels il n'était pas habitué (CARRARI; SOUZA; BEHR, 2017). L'enseignant n'est pas seulement responsable de la transmission des connaissances à ses élèves, car, à ce moment-là, il doit guider le processus d'apprentissage de l'élève, afin qu'il puisse développer ses capacités, c'est-à-dire qu'il doit être aidé à apprendre et donc à développer son autonomie (ROSALIN; CRUZ; MATTOS, 2017). L'enseignant doit accompagner, motiver, dialoguer, être un leader, un médiateur, favoriser et médiatisant une interaction humaine positive (GOULÃO, 2012). On s'attend à ce qu'il soit un modérateur dans les relations interpersonnelles et intrapersonnelles et qu'il auto-évalue continuellement son rendement, en examinant les pratiques.

L'introduction des technologies dans l'environnement d'enseignement est un moyen de stimuler les élèves, en guidant leurs émotions, leurs affections et leurs attitudes (DIAS, 2008). L'enseignant, dans ce nouveau scénario technologique, doit assumer des fonctions qui éveillent la création et l'utilisation des ressources numériques, ce qui implique de revoir ses stratégies d'enseignement, d'améliorer les interactions grâce à la technologie. Pour cette dynamique, il est nécessaire de comprendre les spécificités des canaux qui permettent la communication en ligne synchrone et asynchrone (SALMON, 2000). II faut une bonne structure de communication pour générer une communauté d'apprentissage virtuelle authentique, afin que l'étudiant soit connecté et motivé. Une communication régulière avec les étudiants doit être effectuée à partir de différents canaux de communication, afin qu'ils reconnaissent

RC: 96092

Disponible en: https://www.nucleodoconhecimento.com.br/education-fr/science- 
que la présence de l'enseignant est essentielle. La classe virtuelle « vide » doit être résignée (GOULÃO, 2012).

La salle de classe sans vie, sans présence sociale et cognitive, est un espace « sans vie » (MOREIRA; FERREIRA; ALMEIDA, 2013). Les salles de classe de communication asynchrones virtuelles sont connues sous le nom de « forums ». Ils sont fondamentaux et répondent à l'essence de l'éducation numérique en réseau, car ils ne nécessitent pas une confluence d'enseignants et d'étudiants dans l'espace et le temps, et il y a donc une plus grande flexibilité dans ce modèle éducatif (MOREIRA; HENRIQUES; BARROS, 2020). Pour que ces salles de classe correspondent à un outil de communication puissant, il est crucial qu'elles soient bien organisées et structurées, ce qui nécessite que la communauté connaisse ses règles de fonctionnement et les suive, afin que le virtuel-numérique favorise un apprentissage significatif (SOUZA; ARAGON, 2018). Il est recommandé qu'il y ait des espaces dans cet environnement virtuel pour chaque activité.

Un espace pour la communication des nouvelles et des avertissements, un autre pour répondre aux doutes des élèves, un autre espace informel qui permet l'interaction entre les élèves et les enseignants de manière plus détendue et des espaces créés pour chaque type d'activité, sont des stratégies nécessaires pour que le numérique soit efficace (MOREIRA; HENRIQUES; BARROS, 2020). II existe un certain nombre d'avantages promus par les technologies numériques, mais la formation continue, c'est-à-dire la poursuite, est cruciale, car, dans ce domaine, l'innovation et l'intégration de nouvelles techniques se produisent tout le temps, ce qui nécessite des changements importants dans la pratique de l'enseignement (MOREIRA; HENRIQUES; BARROS, 2020). En ce sens, il est nécessaire de connaître le logiciel, de comprendre ce qui est prévu avec son utilisation, du point de vue pédagogique, et de comprendre si cette ressource numérique spécifique est la plus appropriée (CARRARI; SOUZA; BEHR, 2017).

RC: 96092

Disponible en: https://www.nucleodoconhecimento.com.br/education-fr/science- 
En effet, la simple utilisation des interfaces numériques ne garantit pas en soi des avancées ou des innovations dans les pratiques éducatives (SOUZA; ARAGON, 2018). Plusieurs interfaces Web sociales sont sous-utilisées lorsque les références adoptées reproduisent encore les pratiques acquises dans le Web 1.0. Pour ces raisons, et, en outre, comprendre l'éducation numérique en réseau comme un processus caractérisé par l'utilisation des technologies numériques du Web social, il est nécessaire de promouvoir des pratiques pédagogiques et didactiques actives et constructives (ROSALIN; CRUZ; MATTOS, 2017). Les connaissances et l'apprentissage doivent être développés en collaboration. L'éducation en réseau, en raison de son essence propre, est un processus qui nécessite l'implication profonde des différents acteurs impliqués dans cette articulation, en particulier la définition des objectifs et des parcours d'apprentissage de la communauté.

Le processus a également des répercussions sur les relations étroites construites, en collaboration, entre les pairs qui soutiennent les processus d'innovation et de création de connaissances à partir de voies numériques (MOREIRA; HENRIQUES; BARROS, 2020). Pour la construction collective de ces nouvelles connaissances, il a été de plus en plus courant et rapide la croissance et l'expansion de l'utilisation des ressources éducatives libres (REL). Ceux-ci ont favorisé l'accès et l'utilisation gratuite du contenu et des technologies (GOULÃO, 2012). Ces ressources correspondent à une source inépuisable de ressources numériques qui peuvent être liées à des activités visant à la présentation et à l'enrichissement de contenus. II existe des plateformes qui permettent différentes formes d'approche de la connaissance (plateformes qui intègrent la vidéo, l'audio et l'image, comme YouTube, et d'autres réseaux/médias sociaux et numériques) (SOUZA; ARAGON, 2018).

RC: 96092

Disponible en: https://www.nucleodoconhecimento.com.br/education-fr/science- 


\section{CONNAISSANCES NÉCESSAIRES À L'ENSEIGNEMENT EN} LIGNE

Ce n'est pas le privilège des sciences biologiques de faire face à des défis dans le processus d'enseignement de l'apprentissage, ou même plus, que l'enseignement et la façon d'enseigner. Dans le contexte de l'enseignement en ligne, ces défis n'ont été que plus étendus en exigeant les compétences technologiques sous-développées de l'enseignant. Duré et al. (2018) soulignent que le plus grand défi des enseignants à la fois dans l'enseignement en face à face et l'enseignement en ligne est de rendre les étudiants intéressés par les cours, donc lorsqu'ils recherchent toujours des approches tenant compte de leurs besoins personnels et sociaux, le processus d'enseignement a tendance à être plus efficace. Une autre stratégie qui devrait être adoptée par les enseignants dans ce processus est la grande diversification des mécanismes d'enseignement, tels que les vidéos sur YouTube, les films, les jeux, wanted (OLIVETTI et PEIOTTO, 2014; NICOLA et PANIZ, 2017; ARAÚJO et al., 2011; LEITE et al., 2017; LEÃO et al., 2018)

Tableau 1 - Enseignement des connaissances et des indications pour l'éducation en ligne

Connaissances nécessaires à l'enseignement de la Auteurs

biologie en ligne

Adapter les cours en fonction de la réalité de l'élève, Araújo et al. 2011; qu'elle soit sociale ou personnelle

Leite et al., 2017; Machado, 2017; Leão et al., 2018.

Recherchez des vidéos, des jeux, des applications Araújo et al., 2011; qui peuvent apporter du dynamisme à Leão et al., 2018. l'enseignement de la biologie

Rechercher des méthodes d'évaluation qui Araújo et al., 2011;

RC: 96092

Disponible en: https://www.nucleodoconhecimento.com.br/education-fr/science- 
impliquent l'étudiant, y compris la méthodologie de Leite et al., 2017; Leão groupe, comme la création de vidéos d'exposition et al., 2018.

Carte conceptuelle

Utilisation du quiz (Instagram)

Leão et al., 2018

Recherche dans différents médias en ligne sur les Souza et al., 2020 sujets exposés. Profiter de l'occasion pour développer la pensée critique et l'analyse de 2018 certaines positions en ce moment

Diversification des ressources Youtube, films, jeux, Olivetti et Peiotto, textes 2014; Nicola et Paniz, 2017.

Adaptation de la langue, des lumières et du lieu Soares Filho, 2013. d'enregistrement

Source : Auteur

Souza et al. (2020) ont mené une expérience d'enseignement à travers l'outil « quiz » sur Instagram, comme une sorte de test de connaissances. Les résultats ont montré que les étudiants se sentaient motivés et excités, y compris en développant un mouvement de compétitivité entre eux. En évaluant ce que les élèves ont ressenti à propos de cet outil, le résultat a également été positif, car les étudiants étaient impliqués et motivés. Enfin, la prise en charge de l'enseignant lors de l'enseignement en ligne, de la recherche de la mise à jour de sa langue dans le contexte des élèves et de l'investigation de ses ressources technologiques telles que la lumière et le son, sera certainement faite comme une stratégie dans la réussite de l'enseignement grâce à un meilleur soutien aux élèves (SOARES FILHO, 2013).

La Base nationale commune des programmes d'études (BNCC) souligne la nécessité d'un effort conjoint entre les enseignants, les chercheurs et les organisations nationales afin que les compétences essentielles des étudiants

RC: 96092

Disponible en: https://www.nucleodoconhecimento.com.br/education-fr/science- 
brésiliens de l'éducation de base soient développées, y compris celles liées à l'environnement numérique (FERREIRA et al., 2021). Celles-ci doivent être développées de manière à répondre aux exigences quotidiennes. BNCC présente dix compétences destinées à la formation humaine et intégrale, dont la « culture numérique ». Ainsi, il s'intéresse au développement des compétences et des capacités liées à l'utilisation des technologies numériques en classe, et celles-ci devraient être appropriées à diverses pratiques sociales (MATTAR et al., 2020). Ainsi, la formation continue doit aider les enseignants à comprendre, utiliser et créer les technologies de l'information et de la communication (CHIOSSI; COSTA, 2018).

L'utilisation des technologies numériques devrait être faite de manière critique, significative, réflexive et éthique, en introduisant les pratiques quotidiennes dans le contexte éducatif. L'objectif est de promouvoir la communication, l'accès, la diffusion de l'information, la production de connaissances, la résolution de problèmes, l'exercice du protagonisme et la paternité dans le processus d'interaction avec ces technologies dans le contexte éducatif (LEITE; SILVA, 2017). En ce sens, en comprenant l'intégration des technologies numériques dans la construction du processus d'enseignement-apprentissage et dans la résolution des problèmes quotidiens, le BNCC suggère que les professionnels soient formés pour pouvoir introduire le numérique dans les salles de classe (PIFFERO et al., 2020). Les enseignants de différents niveaux d'éducation et de matières multiples devraient développer des compétences et des compétences qui permettent cette inclusion (CHIOSSI; COSTA, 2018).

Ainsi, les programmes et politiques nationaux qui impliquent l'intégration des technologies dans l'éducation devraient promouvoir des stratégies qui permettent un accès efficace au numérique, mais il existe encore de nombreux obstacles et défis (LEITE; SILVA, 2017). Les technologies de l'information et de la communication doivent aller au-delà de l'utilisation technique et instrumentale du numérique (PIFFERO et al., 2020). Deux facteurs peuvent retarder l'accès au numérique en

RC: 96092

Disponible en: https://www.nucleodoconhecimento.com.br/education-fr/science- 
classe : les conditions physiques et structurelles et une formation inadéquate (FERREIRA et al., 2021). En ce qui concerne la structuration des écoles et la formation des enseignants elle-même, on peut souligner le Programme national de technologie éducative (PROINFO), créé en 1997. Cela a déclenché de nouveaux développements, atteignant le Programme d'innovation et d'éducation connectée. Ces initiatives s'ajoutent à d'autres développées depuis les années 1970 .

L'objectif est de fournir une structure informatique aux écoles afin qu'il soit possible de promouvoir une qualification adéquate des enseignants, rendant possible l'utilisation de TDIC dans le contexte de l'éducation (FERREIRA et al., 2021). Afin d'établir une politique concrète dans le domaine des technologies de l'information afin de tirer parti de l'économie du pays, les technologies sont devenues partie intégrante des contextes les plus divers, mais elles n'ont pas encore été introduites efficacement, car il est encore difficile de mettre en œuvre une culture numérique en classe (CHIOSSI; COSTA, 2018). Cependant, avec la nécessité d'introduire l'enseignement à distance, certaines inégalités ont été mises en évidence en raison de la pandémie (SARAIVA; TRAVERSINI; LOCKMANN, 2020). Même si certaines écoles peuvent promouvoir des moments de rencontre numérique à partir des différentes plateformes, l'accès reste un problème.

En outre, le manque de maîtrise de l'utilisation des plateformes, des réseaux et des médias numériques par l'enseignant rend également difficile l'intégration de la technologie dans l'environnement d'enseignement. La maîtrise de certains outils et technologies implique la connaissance de ces possibilités. De nouvelles technologies émergent et s'améliorent chaque jour, ce qui oblige l'enseignant à rechercher en permanence l'amélioration de ses compétences et compétences liées à la manipulation de telles plateformes et outils numériques (MATTAR et al., 2020). Les connaissances nécessaires à l'exercice d'enseignement imprègnent certains domaines. Ils sont classés dans certaines catégories spécifiques (TARDIF, 2010). Ils sont la connaissance de l'ordre disciplinaire; connaissances professionnelles; les

RC: 96092

Disponible en: https://www.nucleodoconhecimento.com.br/education-fr/science- 
connaissances curriculaires et les connaissances associées au domaine expérimental (MATTAR et al., 2020).

Les compétences technologiques qui seront facturées à l'enseignant dans l'exercice d'enseignement sont liées à certaines connaissances et compétences liées à la manipulation des réseaux et des médias numériques (FERREIRA et al., 2021). En ce sens, afin de rendre l'apprentissage significatif et lié à l'utilisation de la technologie, certaines compétences deviennent primordiales, cependant, pour qu'elles soient exécutées, il est nécessaire que l'enseignant ait les connaissances nécessaires sur leurs possibilités d'utilisation (PIFFERO et al., 2020). La maîtrise des compétences informatiques, des logiciels, des outils, des médias et de divers environnements virtuels (tels que Google Meet et d'autres plateformes) nécessite une certaine littératie et maîtrise du langage numérique (CHIOSSI; COSTA, 2018). Les cours de formation devraient être basés sur cet accent afin que l'enseignement dans l'environnement virtuel favorise un apprentissage efficace, dynamique et attrayant pour les étudiants.

\section{CONSIDÉRATIONS FINALES}

Basée sur l'hypothèse d'apporter quelques indications pour les connaissances de l'enseignant en ce qui concerne l'enseignement en ligne, cette étude était basée sur la méthodologie exploratoire, en recherchant des allusions et des indications dans d'autres études déjà menées. Ainsi, pour élucider les considérations finales de cette étude, la question du guide a été répondue: Comment les enseignants en sciences de la vie peuvent-ils se préparer et quels outils de base devraient-ils connaître pour préparer leurs cours en ligne? L'étude a montré que les enseignants du secondaire et du primaire ont besoin d'utiliser plusieurs outils et stratégies pour préparer leurs cours en ligne. II ne suffit pas d'organiser des cours d'exposition, il est nécessaire d'impliquer les étudiants. L'interaction avec de telles technologies doit cependant

RC: 96092

Disponible en: https://www.nucleodoconhecimento.com.br/education-fr/science- 
être stimulée pour que l'apprentissage ait un sens, il soit nécessaire de maîtriser et de connaître certaines techniques, plateformes, outils, etc.

Certains outils efficaces soulignés par la littérature sont: les cartes conceptuelles, les vidéos YouTube, les films, les jeux, la recherche Google et d'autres réseaux, en bref, les outils qui se développent chez l'étudiant, non seulement dans le contexte de la biologie, mais aussi, qui encouragent l'utilisation consciente et efficace des technologies. En outre, certains soins techniques sont fondamentaux, tels que: l'adéquation de la langue à la classe, compte tenu de son groupe d'âge, de la lumière et de l'audio. En raison du scénario de pandémie et, par conséquent, du manque de classes en classe et de possibilités d'aller sur le terrain, cette étude a apporté certaines limites, qui pourraient être mieux explorées grâce à la recherche avec les enseignants et les étudiants. II est indiqué que d'autres études peuvent être menées de manière appliquée avec les élèves et les enseignants, en étudiant des stratégies et des outils qui s'avèrent efficaces pour l'enseignement de la biologie en ligne au lycée et au primaire.

\section{RÉFÉRENCES}

ARAÚJO, Magnólia Fernandes; SOUSA, Rute Alves; SOUSA, Ivanise Cortez de. Instrumentalização para o Ensino da Biologia. 2 ed. EDFRN, 2011.

BRANDIM, Maria Rejane Lima; NOGUEIRA, Johnson Fernandes. Ensino de Ciências e de Biologia: reflexões e práticas- Parnaíba: Edufpi, 2018.

CARRARO, W. B. W. H.; SOUZA, M.; BEHR, A. Ferramentas de educação a distância utilizadas por profissionais de contabilidade visando a educação continuada. Revista EDaPECI, v. 17, n. 2, p. 144-160, 2017.

CHIOSSI, R. R.; COSTA, C. S. Novas formas de aprender e ensinar: a integração das tecnologias de informação e comunicação (TIC) na formação de professores da

RC: 96092

Disponible en: https://www.nucleodoconhecimento.com.br/education-fr/science- 
educação básica. Texto Livre: Linguagem e Tecnologia, v. 11, n. 2, p. 160-176, 2018.

DIAS, P. Da e-moderação à mediação colaborativa nas comunidades de aprendizagem. Educação, Formação e Tecnologias, v. 1, n. 1, p. 4-10, 2008.

DURÉ, Ravi Cajú; et. al; Ensino De Biologia E Contextualização Do Conteúdo: Quais Temas O Aluno De Ensino Médio Relaciona Com O Seu Cotidiano? Experiências em Ensino de Ciências V.13, No.1, 2018.

FALSARELLA, A. M. Formação continuada e prática de sala de aula: os efeitos da formação continuada na atuação do professor. Campinas, São Paulo: Autores Associados, 2004.

FERREIRA, L. P. S. et al. Autopercepção das competências digitais pelos professores da educação básica sergipana durante o ensino remoto. Scientia Plena Jovem, v. 8, n. 1, 2021.

FERREIRA, T. B.; ALBUQUERQUE, E. B. C.; LEAL, T. F. Formação Continuada de Professores: questões para reflexão. Belo Horizonte: Autêntica, 2007.

GADOTTI, M. Boniteza de um sonho: Ensinar e Aprender com sentido. São Paulo: Editora e Livraria Instituto Paulo Freire, 2008.

GHEDIN, E. Professor Reflexivo: da alienação da técnica à autonomia da crítica. In: PIMENTA, S. G.; GHEDIN, E (Orgs.). Professor reflexivo no Brasil: gênese e crítica de um conceito. $7^{a}$ ed. São Paulo: Cortez, 2012.

GIL, Antonio Carlos Gil. Método de Pesquisa. Ed. Atlas, São Paulo, 2018.

GONÇALVES, Janaína Oliveira. MIRANDA, Maurício Fernando Oliveira de. GONÇALVES JÚNIOR, Edson. Uma reflexão sobre a parceria da família e escola

RC: 96092

Disponible en: https://www.nucleodoconhecimento.com.br/education-fr/science$\underline{\text { biologie }}$ 
em tempos de COVID-19: Aspectos pedagógicos, econômicos e jurídicos. Revista Científica Multidisciplinar Núcleo do Conhecimento. Ano 05, Ed. 11, Vol. 06, pp. 141 154. Novembro de 2020. ISSN: 2448-0959, Link de acesso: https://www.nucleodoconhecimento.com.br/educacao/economicos-e-juridicos, DOI: 10.32749/nucleodoconhecimento.com.br/educacao/economicos-e-juridicos

GOULÃO, M. F. The use of Forums and collaborative learning: A study case. Procedia - Social and Behavioral Sciences, n. 46, p. 672-677, 2012.

JUNGES, F. C.; KETZER, C. M.; OLIVEIRA, V. M. A. de. Formação continuada de professores: saberes ressignificados e práticas docentes transformadas. Educação \& Formação, v. 3, n. 9, p. 88-101, 2018.

LEÃO, Marcelo Franco; et al; Estratégias didáticas voltadas ao ensino das Ciências. Ed. Edibrás. Uberlândia, 2018.

LEITE, M. S.; SILVA, S. R. F. da. Redimensionamento da Computação em Processo de Ensino na Educação Básica: O pensamento Computacional, o Universo e a Cultura Digital. In: VI Congresso Brasileiro de Informática na Educação, 2017.

LEITE, Paula Rayanny Mendonça; et al; O ensino da biologia como uma ferramenta social, crítica e educacional. RECH- Revista Ensino de Ciências e Humanidades Cidadania, Diversidade e Bem-estar. ISSN 2594-8806 - 400. Ano 1, Vol 1, Número 1, Jul-Dez, 2017, p. 400-413

LENZI, Juliana Fernandes de Almeida Castro. SANTOS, Marijara Barbosa Bragato. Sociedade Pós-Moderna Frente À Covid-19: Educadores Em Ação. Revista Científica Multidisciplinar Núcleo do Conhecimento. Ano 06, Ed. 03, Vol. 11, pp. 100 111. Março de 2021. ISSN: 2448-0959, Link de acesso: https://www.nucleodoconhecimento.com.br/educacao/educadores-em-acao

RC: 96092

Disponible en: https://www.nucleodoconhecimento.com.br/education-fr/science$\underline{\text { biologie }}$ 
MACHADO, Claudia Pinto/ Ensino de ciências [recurso eletrônico]: práticas e exercícios para a sala de aula- Caxias do Sul, RS: Educs, 2017.

MATTAR, J. et al. Competências e funções dos tutores online em educação a distância. Educação em Revista, v. 36, 2020.

MOREIRA, J. A.; FERREIRA, A. G.; ALMEIDA, A. C. Comparing communities of inquiry in higher education students: one for all or one for each? OpenPraxis. Internacional Council for Open and Distance Education, v. 5, n. 2, p. 165-178, 2013.

MOREIRA, J. A.; HENRIQUES, S.; BARROS, D. M. V. Transitando de um ensino remoto emergencial para uma educação digital em rede, em tempos de pandemia. Dialogia, n. 34, p. 351-364, 2020.

NICOLA, Jéssica Anese; PANIZ, Catiane Mazocco. A importância da utilização de diferentes recursos didáticos no ensino de biologia. Infor, Inov. Form., Rev. NEaDUnesp, São Paulo, v. 2, n. 1, p.355-381, 2016. ISSN 2525-3476.

OLIVETTI, Maria Madalena de Carvalho; PERIOTTO, Fernando. biologia e as novas tecnologias educacionais, um foco para a educação contemporânea. Revista Eletrônica Científica Inovação e Tecnologia Universidade Tecnológica Federal do Paraná Câmpus Medianeira. Vol.1, p. 9- 2014.

PIFFERO, E. de. L. F. et al. Metodologias Ativas e o ensino de Biologia: desafios e possibilidades no novo Ensino Médio. Ensino \& Pesquisa, v. 18, n. 2, p. 48-63, 2020.

ROCHA, Jéssica. A percepção da ciência pelos alunos do curso de pedagogia UAB/UFMG. V Seminário Internacional de Educação a Distância. UFMG, 2013.

RC: 96092

Disponible en: https://www.nucleodoconhecimento.com.br/education-fr/sciencebiologie 
RODRIGUES, P. M. L.; LIMA, W. dos. S. R.; VIANA, M. A. P. A importância da formação continuada de professores da educação básica: a arte de ensinar e o fazer cotidiano. Saberes Docentes em Ação, v. 3, n. 1, p. 28-47, 2017.

ROSALIN, B. C. M.; CRUZ, J. A. S.; MATTOS, M. B. G. de. A importância do material didático no ensino a distância. Revista on line de Política e Gestão Educacional, v. 21, n. 1, p. 814-830, 2017.

SALMON, G. E-Moderating. The Key to Teaching and Learning Online. London: Kogan Page, 2000.

SARAIVA, K.; TRAVERSINI, C. S.; LOCKMANN, K. A educação emtempos de COVID-19: ensino remoto e exaustão docente. Práxis educativa, v. 15, p. 1-24, 2020.

SOARES FILHO, Daniel. Conteúdos EAD: Pequenos detalhes que fazem a diferença. V Seminário Internacional de Educação a Distância. UFMG, 2013.

SOUZA, Dominique Guimarães; et. al. Redes sociais e o ensino de biologia: $O$ uso do Quis do Instagram como Recurso Didático. Recite - Revista Carioca de Ciência Tecnologia e Educação · December 2020.

SOUZA, S. S. dos. S. de.; ARAGON, G. T. Estilos de aprendizagem e ensino a distância na perspectiva da inclusão. EaD em Foco, v. 8, n. 1, 2018.

TARDIF, M. Saberes docentes e formação profissional. $17^{a}$ ed. Petrópolis, RJ: Vozes, 2010.

TREBIEN, M. M et al. Formação continuada de professores: uma epistemologia da prática. Ambiente: Gestão e Desenvolvimento, v. 13, n. 1, p. 91-102, 2020.

Soumis: Septembre, 2021.

RC: 96092

Disponible en: https://www.nucleodoconhecimento.com.br/education-fr/science$\underline{\text { biologie }}$ 
Approbation: Septembre, 2021.

RC: 96092

Disponible en: https://www.nucleodoconhecimento.com.br/education-fr/science$\underline{\text { biologie }}$ 